\title{
Instrumento de evaluación de un videojuego educativo facilitador del aprendizaje de habilidades prosódicas y comunicativas
}

\section{The evaluation tool of an educational video game oriented to the learning of prosodic and communicative skills}

\author{
Lourdes Aguilar ${ }^{1}$ \\ Universitat Autònoma de Barcelona, Bellaterra, España \\ lourdes.aguilar@uab.cat \\ Ferran Adell ${ }^{2}$ \\ Universitat Oberta de Catalunya, Barcelona, España \\ myrk@uoc.edu
}

\begin{abstract}
Resumen
El artículo dirige la mirada al procedimiento de evaluación que acompaña al videojuego educativo Pradia: misterio en la ciudad. El videojuego está concebido como herramienta de apoyo educativo para la mejora de las habilidades prosódicas y comunicativas de las personas con Síndrome de Down, pero para que sea eficaz es preciso que lo acompañe un método de exportación de los datos y de evaluación de los resultados. Para dar respuesta a esta necesidad, el Test de habilidades prosódicas del videojuego educativo PRADIA: Misterio en la ciudad (THAP-PRADIA) analiza el grado de éxito en cada una de las actividades resueltas en el videojuego y ofrece unas puntuaciones que estiman el progreso en la curva de aprendizaje. Los informes de resultados exponen distintos niveles de detalle: las puntuaciones pueden agruparse de acuerdo con los objetivos de aprendizaje que persigue el videojuego, o bien puede extraerse un informe para cada una de las dinámicas del juego. La organización de los resultados de lo más general a lo más particular permite obtener una valoración global de la actuación comunicativa y de las destrezas prosódicas del usuario, a la vez que observar las carencias más significativas, con el fin último de proponer nuevas estrategias didácticas.
\end{abstract}

Palabras clave: aprendizaje en entornos virtuales, evaluación educativa de videojuegos, desarrollo de competencias prosódicas, desarrollo de competencias comunicativas, recursos para necesidades educativas especiales.

\begin{abstract}
The article focuses on the evaluation procedure that accompanies the educational video game Pradia: mystery in the city. The video game is designed as an educational support tool that aims to improve the prosodic and communication skills of people with Down Syndrome, but to be effective it must be accompanied by a method of data export and evaluation of the results. To do this, the Test of Prosodic Skills of PRADIA (THAPPRADIA) analyzes the degree of success in each of the activities included in the game and offers scores that estimate the learning progress. The exportation of the data and its latest analysis allow to obtain reports of results with different levels of detail: the scores can be grouped according to the learning objectives pursued by the video game, or a report can be extracted for each of the dynamics of the game. The organization of the results from the most general to the more particular allows us to obtain a global assessment of the communicative performance and the prosodic skills of the user throughout the game time,
\end{abstract}

${ }^{1}$ ORCID identifier: 0000-0003-4619-9046

${ }^{2}$ ORCID identifier: 0000-0002-4192-3325 
and at the same time to observe the most significant shortcomings, with the goal of proposing new teaching strategies.

Keywords learning in virtual environments, educational evaluation of video games, development of prosodic skills, development of communicative skills, special needs learning tools.

\section{Introducción}

El uso de actividades lúdicas en provecho de los procesos educativos está presente a lo largo de la historia (Huizinga, 1984): entre otras ventajas, merecen especial interés el aumento del interés en la actividad y de la participación activa, de la aceptación de normas o de la tolerancia a la frustración. En este contexto y dada la importancia creciente de la implantación de las Tecnologías de la Información y la Comunicación (TIC) en la educación actual, el uso del videojuego como herramienta didáctica ha adquirido gran relevancia.

Una de las principales motivaciones para avanzar en este marco de trabajo es que los videojuegos permiten definir un entorno de juego, atractivo en sí mismo para la mayoría de los individuos, en el que el aprendizaje está integrado en el guion narrativo, de modo que el jugador adquiere e interioriza la nueva información que se le está ofreciendo de forma desapercibida. Las principales potencialidades educativas de los videojuegos emanan de lo que se conoce como inmersión (es decir, la capacidad que tiene el sistema de provocar en el usuario una identificación con su personaje y el mundo virtual creado en el juego). Esta característica de los videojuegos es especialmente útil para mantener la atención del usuario en lo que sucede en el juego, asegurando que, a diferencia de otros medios digitales en que se necesita la intención o necesidad de aprender, el interés del jugador se basa, primero, en las ganas de empezar a jugar, y después, en el placer de seguir jugando para resolver algún enigma o acometer alguna hazaña. Si, además, como es el caso de la práctica educativa que describe este estudio, el público objetivo es el alumnado con Necesidades Específicas de Apoyo Educativo derivadas de la discapacidad, es determinante definir un ambiente en que el garante de la concentración en la actividad sea el propio juego: en breve, necesitamos una historia interesante e inmersiva, implementada en un entorno de juego sencillo, pero que permita tomar decisiones al jugador.

En este marco de investigación, considerando el videojuego educativo una estrategia facilitadora del aprendizaje significativo, proponemos una herramienta dirigida a mejorar la competencia comunicativa de las personas con Síndrome de Down (en adelante, SD), con el fin último de que consigan mayores cotas de autonomía personal e integración social. En este sentido, la propuesta educativa tiene como propósito alcanzar el dominio de unas estrategias prosódicas que capaciten al alumnado para, entre otros objetivos, relacionar las formas lingüísticas con las funciones comunicativas que demanda cada situación social; participar en relaciones interpersonales propias de las circunstancias en las que se desenvuelva su vida; diferenciar contenidos en función del propósito que se persigue; manifestar informaciones sobre sí mismo y sus necesidades, así como interpretar el medio social y el comportamiento de los demás.

Instrumento de evaluación de un videojuego educativo facilitador del aprendizaje de habilidades prosódicas y comunicativas. Lourdes Aguilar y Ferran Adell 
En lo que sigue, el artículo, una vez acotada la influencia que ejerce la prosodia en el dominio de las habilidades comunicativas, pasa a exponer las principales características del videojuego educativo Pradia: misterio en la ciudad, y el diseño y desarrollo de una herramienta informática post-videojuego que permite evaluar el progreso en el aprendizaje: el Test de habilidades prosódicas del videojuego educativo PRADIA: Misterio en la ciudad (THAP-PRADIA).

\section{El papel de la prosodia en el desarrollo de la competencia comunicativa}

Cuando producimos un enunciado, no solo articulamos un sonido tras otro, sino que también variamos la intensidad, el tono de voz y la velocidad de habla, entre otras posibles modificaciones. Por ello, podemos interpretar de manera radicalmente distinta frases con la misma secuencia de sonidos ("Está aquí." pronunciado en una gama emocional desde la alegría hasta la tristeza, pasando por el enfado o la sorpresa). Las propiedades de las que hablamos son los rasgos prosódicos (o suprasegmentales), que actúan sobre grupos de sonidos (sílaba, palabra o frase), no sobre sonidos aislados, y que son inherentes a la oralidad (difícilmente representables en la escritura) (Aguilar et al., 2009). Desde el marco de la fonología entonativa (Ladd, 2008), los fenómenos prosódicos pueden explicarse según tres categorías: acento (con distintos grados de prominencia o fuerza acentual), entonación (con especial referencia a la modalidad oracional) y organización prosódica (agrupación rítmica de los enunciados).

Al ser un fenómeno a la vez de forma y significado, las diferencias prosódicas inciden en los procesos interpretativos, hasta el punto de no es posible captar la función comunicativa de un enunciado hasta que no disponemos de dicha información, lo que resulta en graves problemas de interacción social en las personas con SD. Para empezar, una consecuencia de los fallos prosódicos es que entorpecen la inteligibilidad de los enunciados, ya de por sí dañada en el habla de las personas con SD (Kumin, 2002). Además, en situaciones cotidianas, el hablante puede provocar malentendidos en frases con entonaciones ambiguas o incluso llegar a ofender involuntariamente a su interlocutor por el mero hecho de emplear una entonación inapropiada. Un enunciado del tipo "Puede recoger su coche a partir de las cuatro" pronunciado con una entonación propia de una orden (tempo rápido, elevación del tono y de la intensidad, uso de un registro tonal agudo) ocasiona que el destinatario lo interprete como "Tiene que recoger su coche a partir de las cuatro". También en el ámbito de la comprensión se encuentran dificultades motivadas por fenómenos prosódicos: por ejemplo, cuando la persona con SD no contesta a una pregunta porque no ha logrado percibir la entonación interrogativa, con un típico final ascendente.

Podemos afirmar, por tanto, que con una prosodia apropiada se minimizan los fracasos comunicativos, que pueden dar lugar a inseguridades y a una baja autoestima. En este sentido, formulamos la hipótesis de que la adquisición de las adecuadas estructuras prosódicas en relación con los distintos sistemas de conocimiento gramatical, sociolingüístico, discursivo y estratégico es fundamental para el desarrollo de las habilidades intra e interpersonales, al lograr una adecuada regulación de los hechos sociales y emocionales (Canale, 1983).

Instrumento de evaluación de un videojuego educativo facilitador del aprendizaje de habilidades prosódicas y comunicativas. Lourdes Aguilar y Ferran Adell

Página 3 de 16 
Ahora bien, para que las personas con SD lleguen a dominar los matices en el ritmo, la cadencia o la melodía es esencial que los practiquen junto con los demás componentes del lenguaje y en contextos reales, ya que el significado del componente prosódico tiene múltiples variaciones y se adapta a las situaciones comunicativas. Por eso, en el videojuego educativo Pradia: misterio en la ciudad todas las acciones relacionadas con la prosodia se desarrollan en la trama del juego para lograr un aprendizaje significativo, de modo que el individuo pueda trasladar los contenidos adquiridos a su vida real y mejore sus habilidades comunicativas.

La identificación de los objetivos de aprendizaje que incluye el videojuego se ha basado en los déficits prosódicos y pragmáticos documentados en estudios previos en el alumnado con SD. Aunque el comportamiento lingüístico de las personas con SD es heterogéneo, dado que presentan una gran variedad de perfiles de desarrollo cognitivo y enfermedades asociadas, como trastornos de la visión y de la audición (Rice et al., 2005; Kent \& Vorperian, 2013), en lo que respecta al componente prosódico se han podido relacionar algunos de los errores de habla con dificultades en la identificación de las fronteras entre las palabras y las frases (Heselwood, Bray \& Crookston, 1995), así como se han señalado mejores capacidades de los niños con SD en el ámbito de la comprensión que en el de la producción, y en ciertas tareas en las que pueden apoyarse en el significado (Stojanovik, 2011).

\section{Decálogo de un videojuego educativo para mejorar la prosodia de las personas con SD}

Pradia: misterio en la ciudad (www.pradia.net) es un videojuego educativo que se basa en la arquitectura del prototipo de videojuego La piedra mágica, diseñado para personas con diversidad funcional y distribuido bajo un tipo de licencia creative commons que no permite el uso comercial ${ }^{3}$ (González-Ferreras et al., 2017). Destacamos a continuación los aspectos más relevantes de la nueva herramienta:

(1) El videojuego educativo Pradia pertenece al género de la aventura gráfica. La aventura gráfica, a diferencia de las baterías de ejercicios o actividades multimedia, permite un proceso de inmersión del jugador en el mundo virtual mostrado, provocando una identificación del individuo con su personaje, lo que aumenta la implicación del usuario en la resolución de la historia. Este hecho, a la vez, favorece que la persona con necesidades específicas de apoyo educativo derivadas de la discapacidad interiorice los contenidos de aprendizaje y los traslade a su vida diaria.

(2) El jugador elige su avatar. Con el objetivo de potenciar su capacidad para integrarse en el mundo creado por el juego y de sentirse involucrado en la narración, el jugador

\footnotetext{
${ }^{3}$ En concreto, la licencia incluye las siguientes condiciones: Reconocimiento - No comercial - Compartir igual: El autor permite copiar, reproducir, distribuir, comunicar públicamente la obra, y generar obras derivadas siempre y cuando se cite y reconozca al autor original. La distribución de las obras derivadas deberá hacerse bajo una licencia del mismo tipo. No se permite utilizar la obra con fines comerciales.
}

Instrumento de evaluación de un videojuego educativo facilitador del aprendizaje de habilidades prosódicas y comunicativas. Lourdes Aguilar y Ferran Adell 
tiene la oportunidad de elegir su propio avatar, ya que es un factor necesario para la identificación entre la persona y el jugador ${ }^{4}$.

(3) El videojuego educativo Pradia no es una herramienta de aprendizaje autónomo. El videojuego no se ha concebido para su uso como recurso de autoaprendizaje. Las sesiones deben realizarse con el apoyo de un profesional o acompañante externo que guíe al jugador con discapacidad a lo largo de las sesiones de juego y que contribuya de manera personalizada a la calidad de la experiencia lúdica. La tarea de este acompañante (educador, tutor, familiar) consiste en brindarle diferentes tipos de apoyo según su interrelación con el sistema. El tipo de refuerzo viene condicionado por las habilidades individuales de los jugadores, de manera que no puede delimitarse de antemano, sino que lo define el acompañante teniendo en cuenta las necesidades, intereses y deseos de cada alumno.

(4) El jugador cuenta con un asistente virtual. Para preservar en todo lo posible el carácter lúdico de la aplicación (minimizando en todo lo posible la intervención del acompañante externo), se ha incluido un asistente virtual, en forma de mascota, que se mantiene al lado del jugador en todo momento y, que, además de guiarle en la consecución de los retos de la aventura, le ofrece pistas, explicaciones o aclaraciones en situaciones de error, bloqueo o pérdida de la atención. La mascota tiene características mágicas: dotado de la capacidad del lenguaje, acompaña al héroe de la aventura gráfica durante todo el camino y sabe perfectamente cuándo tiene que intervenir para ayudar al jugador; reconoce las reacciones de los personajes y ayuda al jugador a interpretarlas adecuadamente; además, tiene buenos modales y sabe comportarse en diferentes ámbitos sociales, lo que refuerza en el videojuego el componente de aprendizaje observacional del entorno. La existencia de este asistente virtual, junto con la posibilidad de seleccionar diferentes niveles de dificultad de la aventura gráfica, son indispensables para adaptar el desarrollo de la experiencia de juego al alto grado de heterogeneidad que existe en las competencias lingüísticas de las personas con SD.

(5) El fin educativo del videojuego es la mejora de la producción y la percepción de la prosodia con efectos comunicativos. Con el fin de no separar el proceso de aprendizaje de la experiencia de juego, todas las actividades se integran en el guion narrativo: para avanzar en su aventura, el héroe debe comunicarse con el resto de los personajes no jugadores en distintos contextos sociales, donde se le plantean retos relacionados con las habilidades prosódicas, desde el punto de vista de la expresión oral y la comprensión. Las categorías que se incluyen como objetivos de aprendizaje son las siguientes (Aguilar y Gutiérrez-González, en proceso de revisión):

—Acentuación enfática (o prominencia acentual)

-Agrupación rítmica de los enunciados, en particular, la organización en grupos prosódicos relacionada con la estructura sintáctica.

\footnotetext{
${ }^{4}$ Aunque los recursos disponibles han impedido un desarrollo complejo de la personalización del avatar no hemos querido dejar de ofrecer a los usuarios pequeñas modificaciones en la apariencia del héroe para favorecer este proceso de identificación.
}

Instrumento de evaluación de un videojuego educativo facilitador del aprendizaje de habilidades prosódicas y comunicativas. Lourdes Aguilar y Ferran Adell 
—Entonación, con especial énfasis en la adecuada relación entre forma y significado en el uso de las modalidades oracionales.

—Actos de habla corteses (saludos, despedidas, ofrecer, dar las gracias).

—Expresión lingüística de emociones básicas (sorpresa, alegría, enfado, tristeza).

(6) El videojuego permite trabajar habilidades distintas a las prosódicas. Además de las actividades planteadas para desarrollar la competencia prosódica, el videojuego incluye un conjunto de desafíos puramente lúdicos, para conseguir un entorno de juego inmersivo a través de un relato que provoque que el jugador quiera formar parte de él y cuyo recorrido dependa directamente de las decisiones que este tome. Se trata, entre otras, de actividades relacionadas con habilidades motrices (como la resolución de un rompecabezas que permite obtener el nombre de uno de los personajes clave en la resolución de la historia), o con el desarrollo de hábitos sociales y personales sostenibles (como la necesidad de que el jugador recuerde apagar las luces al salir de casa).

(7) Las actividades de percepción de la prosodia ejercitan la discriminación basada únicamente en diferencias prosódicas. Para mejorar en la identificación e interpretación de las estructuras prosódicas, el videojuego incluye a lo largo de la aventura y de manera integrada en el guion narrativo actividades de discriminación auditiva entre pares mínimos (dos oraciones que coinciden en todos los fonemas y solo se diferencian en una categoría suprasegmental).

(8) En las actividades de producción de la prosodia el jugador practica con su propia voz mediante la lectura, imitación o producción guiada de los enunciados. El jugador se enfrenta a obstáculos cuya resolución depende de que produzca oralmente las estructuras prosódicas comunicativamente adecuadas en el contexto.

(9) El éxito en la resolución de las actividades de percepción se evalúa de forma automática por el sistema. Dado que las dinámicas son de selección entre dos opciones, en las actividades de percepción el sistema calcula el resultado de forma automática, y el jugador obtiene una retroalimentación que le indica si está actuando de forma correcta.

(10) El acompañante externo juzga el éxito en la resolución de las actividades de producción. En las actividades de producción, dada la variabilidad en la producción de un enunciado (con mayor o menor inteligibilidad, velocidad de elocución, intensidad, número de pausas, etc.) y las limitaciones fonéticas del habla de las personas con SD, es preciso que sea el acompañante externo quien valore el grado de ajuste a lo pedido de la respuesta. Ahora bien, con el fin de preservar el componente lúdico, la evaluación se hace mediante un teclado distinto al usado para el videojuego, de modo que los juicios pasan desapercibidos para el jugador. Del mismo modo que en las actividades de percepción, el jugador oye un mensaje del propio videojuego que le indica en qué medida ha logrado el objetivo del reto.

Instrumento de evaluación de un videojuego educativo facilitador del aprendizaje de habilidades prosódicas y comunicativas. Lourdes Aguilar y Ferran Adell 


\section{Evaluación del aprendizaje}

Si es bien conocido el interés de la comunidad educativa por utilizar videojuegos en las aulas, la mayoría de la investigación en juegos formativos ha centrado su objeto de estudio en la dimensión tecnológica, relegando a un segundo plano a la educativa (del Moral et al., 2012; Rodríguez-Hoyos y João Gomes, 2013). Así pues, aunque los beneficios que tiene el uso de videojuegos en los dominios cognitivo, motivacional, emocional y social parecen demostrados (García Pernía et al., 2011; Wuang et al., 2011; Revuelta Domínguez y Guerra Antequera, 2012; Granic et al., 2014: Alloza-Castillo et al., 2017), en entornos didácticos resulta complejo comprobar empíricamente que la estimulación que generan tenga un impacto directo en el progreso del aprendizaje.

En este contexto, para que el videojuego educativo PRADIA: Misterio en la ciudad sea eficaz es imprescindible que lo acompañe un método de exportación de los datos y de evaluación de los resultados. Por ello, se ha desarrollado el Test de habilidades prosódicas del videojuego educativo PRADIA: Misterio en la ciudad (THAP-PRADIA), que analiza los resultados de la actuación del jugador en cada una de las actividades resueltas en el videojuego y ofrece unas puntuaciones en las categorías que son objeto de aprendizaje. El modelo que seguimos es el "dashboard estratégico" donde es preciso definir el número de indicadores de la actuación (en este caso, pragmática y prosódica) y transformar la información a un lenguaje fácilmente interpretable por parte de los agentes educativos.

\section{Procedimiento}

Con el fin de cumplir los objetivos de aprendizaje, el videojuego incluye un catálogo de actividades o dinámicas (tipos específicos de interacciones que el jugador establece con el mundo virtual en relación con unas áreas de aprendizaje que se desglosan en una serie de saberes procedimentales y conceptuales. El THAP-PRADIA se basa en asociar cada una de las actividades con los contenidos, de manera que al final del videojuego se confecciona un informe de los aciertos/errores en cada una de las áreas con el cometido de que el educador proponga un plan de actividades suplementarias de refuerzo en las áreas más afectadas.

Los principales objetivos de aprendizaje del videojuego giran en torno a la competencia prosódica, lingüística emocional y pragmática, aunque es preciso señalar que el tratamiento aislado de los fenómenos prosódicos es puramente una estrategia metodológica, dado que es bien sabido que son interdependientes: a modo de ejemplo, las exclamaciones son una modalidad entonativa que expresa una carga emocional en relación con el contexto comunicativo. Tampoco obedece la organización a ningún tipo de orden ni jerárquico ni en el tratamiento de los contenidos, por lo que no debe entenderse como una propuesta de secuenciación pedagógica.

Como hemos dicho, aunque el fin educativo del videojuego es la mejora de las destrezas prosódicas con efectos comunicativos, los desafíos lúdicos añaden otros aspectos como el aumento de vocabulario comprensivo y de vocabulario productivo, entre otras habilidades. En estos casos, se trata de objetivos transversales que esperamos alcanzar de manera indirecta mediante la interacción de los jugadores con el videojuego.

Instrumento de evaluación de un videojuego educativo facilitador del aprendizaje de habilidades prosódicas y comunicativas. Lourdes Aguilar y Ferran Adell 


\section{Áreas de aprendizaje principales}

Las áreas de aprendizaje principales atañen a tres componentes de la competencia comunicativa (definida como la capacidad de una persona para comportarse de manera eficaz y adecuada en una determinada comunidad de habla, lo que implica respetar tanto las reglas lingüísticas como las normas de uso, relacionadas con el contexto sociohistórico y cultural en el que tiene lugar la comunicación): competencia prosódica; competencia linguística emocional y competencia pragmática. Las figuras 1,2 y 3 ilustran los contenidos que quedan explicitados en (1), (2) y (3), con las definiciones adaptadas del Diccionario de términos clave de ELE, Centro Virtual Cervantes.

(1) CoMPETEnCIA PROSÓDICA: Capacidad, como oyente y hablante, de distinguir y producir las estructuras prosódicas adecuadas a los significados lingüísticos asociados.

(1.1) Las categorías que permiten evaluar la competencia prosódica son:

(1.1.1) Modalidad oracional: Estructuras que caracterizan la forma de comunicación con el interlocutor: enunciativa, interrogativa, exclamativa e imperativa.

(1.1.2) Agrupación rítmica de los enunciados: Organización en partes distintivas y significativas de una corriente continua de sonido.

(1.1.3) Acentuación enfática: Propiedad de las palabras o partes de las palabras que destacan en el enunciado por su fuerza acentual.

(1.2) La competencia prosódica se aborda desde los ámbitos de la percepción y la producción:

(1.2.1) Discriminación auditiva de estructuras prosódicas: Capacidad, como oyente, de distinguir entre categorías prosódicas (acentuación enfática, agrupación rítimica, modalidad oracional).

(1.2.2) Expresión oral de estructuras prosódicas: Capacidad, como hablante, de producir las categorías prosódicas (acentuación enfática, agrupación rítmica, modalidad oracional).

(2) COMPETENCIA LINGÜÍsTICA EMOCIONAL: Habilidad para reconocer las emociones ajenas expresadas en los enunciados y para expresar oralmente las propias emociones.

La competencia emocional se aborda desde los ámbitos de la percepción y la producción:

(2.1) Identificación de emociones en los enunciados: Destreza de discriminación auditiva de emociones.

(2.2) Expresión oral de emociones: Destreza de expresión oral de emociones.

(3) COMPETENCIA PRAGMÁTICA: Capacidad de desempeñar un uso comunicativo de la lengua en el que se tengan presentes no solo las relaciones que se dan entre los signos lingüísticos y sus referentes, sino también las relaciones que se dan entre el sistema de la lengua, por un lado, y los interlocutores y el contexto de comunicación, por otro. 
La competencia pragmática se concreta en una selección de actos de habla y de manifestaciones de cortesía verbal.

(3.1) Actos de habla: Unidad básica de la comunicación lingüística con la que se realiza una acción (saludo, despedida...)

La manifestación prosódica de los actos de habla seleccionados se aborda desde los ámbitos de la percepción y la producción:

(3.1.1) Identificación de acto de habla: Destreza de reconocer los enunciados que realizan una acción.

(3.1.2) Producción de acto de habla: Destreza de expresar oralmente enunciados que realizan una acción.

(3.2) Cortesía verbal: Conjunto de estrategias lingüísticas destinadas a favorecer la interacción entre los hablantes.

La manifestación de la cortesía verbal se aborda desde los ámbitos de la percepción y la producción:

(3.2.1) Identificación de cortesía verbal: Destreza de reconocer los enunciados que apoyan la cortesía.

(3.2.2) Producción de cortesía verbal: Destreza de expresar oralmente enunciados que apoyan la cortesía.

Para integrar las tareas de expresión oral (de categorías prosódicas, emociones, actos de habla y cortesía verbal) en el guion narrativo del videojuego se le plantean al jugador diferentes retos que tienen que ver con la lectura de un enunciado, la imitación (repetición de un modelo previo), el habla guiada (enunciado conseguido a partir de las indicaciones de alguno de los personajes no jugadores o del asistente virtual), o el habla espontánea (enunciado obtenido sin instrucciones previas).

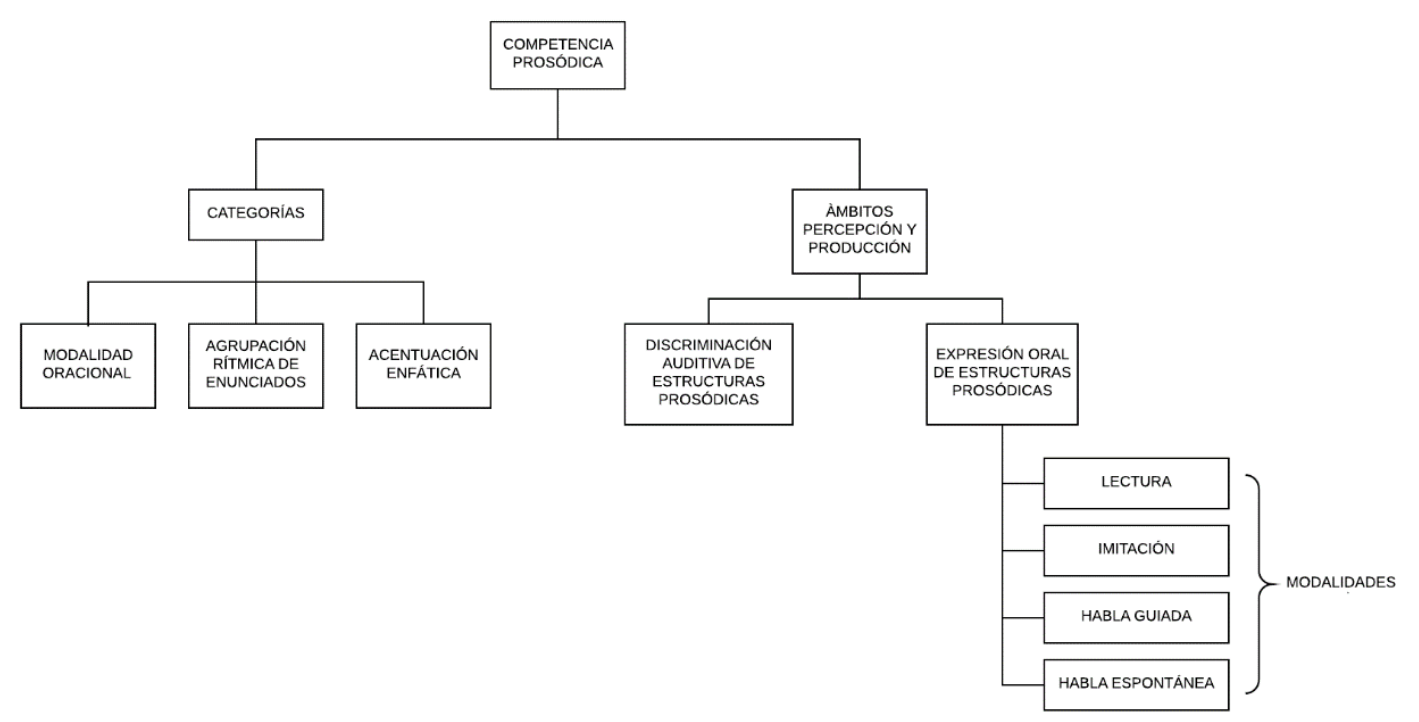

Figura 1. Mapa conceptual del área de aprendizaje de la competencia prosódica. 


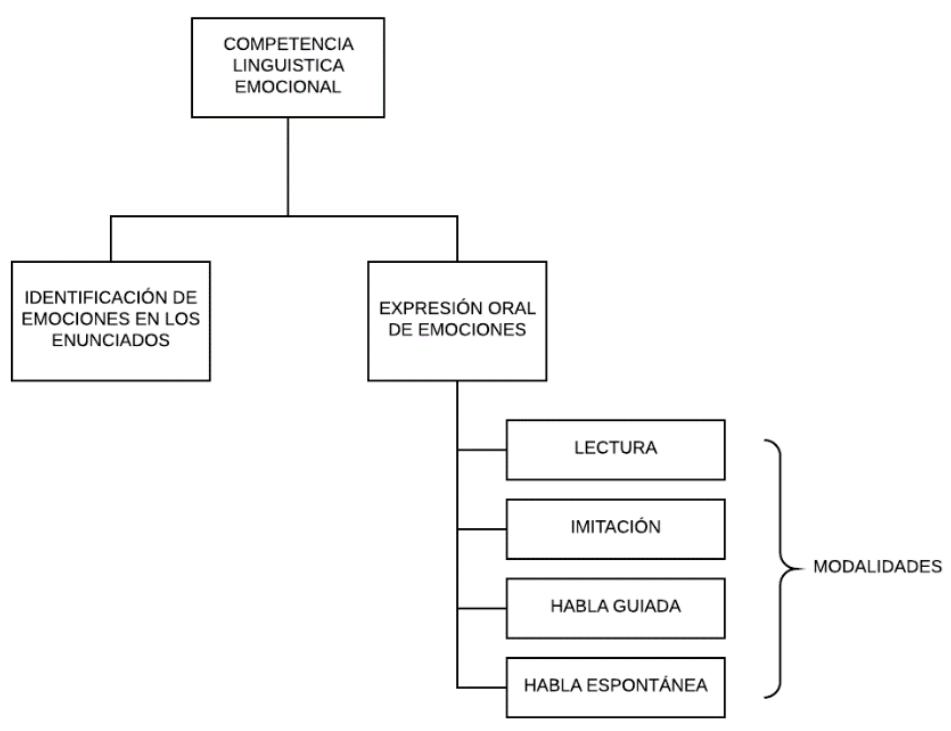

Figura 2. Mapa conceptual del área de aprendizaje de la competencia lingüística emocional.

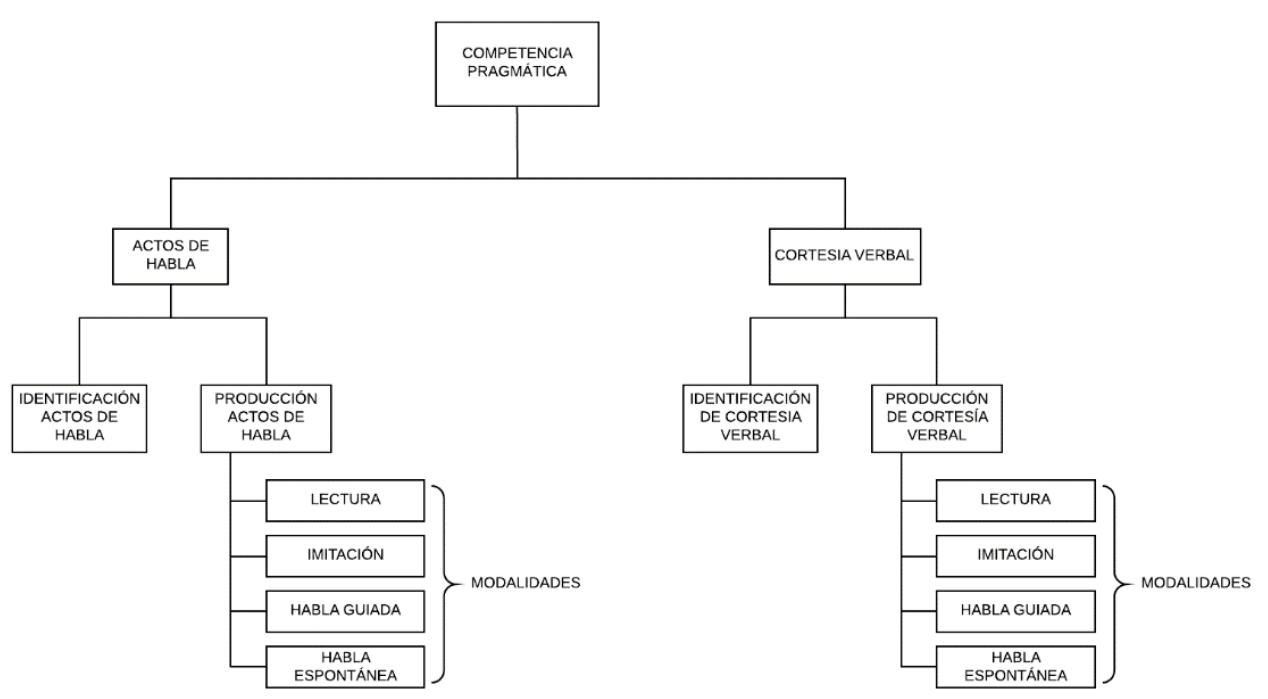

Figura 3. Mapa conceptual del área de aprendizaje de la competencia pragmática.

\section{Áreas de aprendizaje genérico}

Para dar cuenta del aprendizaje indirecto que alcanza el jugador por el simple hecho de estar inmerso en el ecosistema del videojuego, donde existe una comunidad de personajes cuyos procesos vitales se relacionan entre sí y se desarrollan en función de las circunstancias ambientales, identificamos unas áreas de aprendizaje de tipo genérico, 
según define la serie (4) - (10). Aunque es preciso refinar la clasificación usada hasta el momento para incluir destrezas relacionadas con el conocimiento del medio natural, social y cultural (interacción individuo-sociedad, medio físico-medio social, entre otros ítems, véase del Moral y Villalustre, 2012 para una propuesta de instrumento de evaluación), en el estado actual de desarrollo la herramienta THAP-PRADIA registra áreas de aprendizaje que comprenden habilidades léxicas y discursivas (competencia léxica; comprensión de la narración; memoria de la narración) y otras áreas de aprendizaje que vienen dadas por las características propias de los videojuegos (competencia en actividades lúdicas; coordinación oculo-manual; percepción espacial; atención).

(4) COMPETEnCIA LÉXICA: Capacidad de entender y de utilizar unidades léxicas (palabras y otras unidades mayores formadas por dos o más palabras con un sentido unitario) de la lengua.

(4.1) Comprensión de vocabulario: Destreza de entender el conjunto de unidades léxicas de la lengua.

(4.2) Producción de vocabulario: Capacidad de utilizar unidades léxicas de la lengua.

(5) COMPRENSIÓN DE LA NARRACIÓN: Capacidad comunicativa que rebasa el plano estrictamente lingüístico y abarca el proceso completo de interpretación de la historia del videojuego.

(6) MEMORIA DE LA NARRACIÓN: Capacidad de almacenar, analizar e interpretar la información sobre la historia que narra el videojuego.

(7) COMPETENCIA EN ACTIVIDADES LÚDiCAS: Capacidad de resolver problemas en situaciones de juego (rompecabezas...)

(8) CoORdinación oculo-MAnUal: Capacidad de coordinar las manos y dedos, en coordinación con los ojos.

(9) PERCEPCIÓN ESPACIAL: Capacidad de evaluar cómo están situados los objetos en el espacio e investigar sus relaciones con el entorno.

(10) AtEnción: Capacidad para concentrar la mirada en uno o varios aspectos de la realidad y prescindir de los restantes.

\section{Indicadores}

La herramienta THAP-PRADIA asigna una puntuación numérica a cada una de las actividades, de acuerdo con los indicadores de logros en el aprendizaje descritos en la serie (11-13), que varían según la tipología de las dinámicas:

(11) Dinámicas de percepción (discriminación auditiva de estructuras prosódicas): Son indicadores: tiempo de reacción, número de intentos fallidos, número de veces en que el jugador recurre a la repetición de los enunciados.

Instrumento de evaluación de un videojuego educativo facilitador del aprendizaje de habilidades prosódicas y comunicativas. Lourdes Aguilar y Ferran Adell 
(12) Dinámicas de producción (expresión oral de estructuras prosódicas): Se parte de la premisa de que es el acompañante externo quien juzga la adecuación de las respuestas de voz de los hablantes con SD, dado que los métodos automáticos de diagnosis son poco eficaces en un tipo de habla con una gran variabilidad en la inteligibilidad, velocidad de elocución, intensidad, número de pausas, etc. y con serias limitaciones fonéticas (Kumin, 2002; Martin et al., 2009). Para no añadir complejidad a la tarea de valoración, el juicio se emite en una escala discreta 1-2-3, donde 1 corresponde a "resolución fallida", 2 a "resolución satisfactoria" y 3 a "resolución excelente". Una vez exportados, los valores se transforman a una escala numérica 0-10, más fácilmente interpretable por parte de los agentes educativos dada su mayor tradición.

(13) Dinámicas de juego. Son indicadores: tiempo empleado en la resolución de la actividad, número de intentos fallidos (número de piezas mal colocadas en el caso de un puzzle).

\section{Informes de resultados}

La exportación de los datos obtenidos por el jugador en su tiempo de juego y su posterior análisis permiten obtener informes de resultados con distintos niveles de detalle: las puntuaciones pueden agruparse siguiendo las categorías que encabezan cada uno de los mapas conceptuales de las figuras 1-3, como ilustra la figura 4, o bien puede extraerse un informe para cada una de las dinámicas del juego, como muestra la serie (14) - (16). Esta organización de los resultados en diferentes dimensiones, de lo más general a lo más particular, permite obtener una valoración global de la actuación comunicativa y de las destrezas prosódicas del usuario a lo largo del tiempo de juego, a la vez que observar las carencias más significativas en las áreas en las que ha alcanzado resultados poco satisfactorios.

El informe global de resultados de la figura 4 refleja puntuaciones que se sitúan por encima del 7 en todos los ámbitos de análisis: podemos decir, por tanto, que se trata de un hablante con SD con un dominio notable de las habilidades prosódicas con efectos comunicativos. Ahora bien, resultan interesantes las diferencias que se observan en los planos de la comprensión y la producción: de forma clara, los resultados son mejores en las tareas de discriminación auditiva de estructuras prosódicas que en las tareas de expresión oral de estructuras prosódicas, en la línea de lo hallado en el experimento de Stojanovik (2011). También se desprende de las puntuaciones obtenidas en las categorías prosódicas que la mayor dificultad recae en la agrupación rítmica de enunciados (un déficit común en el habla con SD, según documenta el estudio de Kent y Vorperian, 2013). Por último, si comparamos el grado de éxito de las dinámicas dependiendo del modo de producción que se le requiere al jugador, no parece haber diferencias relevantes, aunque sí una tendencia a peores resultados conforme se pasa de la lectura a la imitación y el habla guiada. Esta tendencia es especialmente significativa ya que en las actividades de habla guiada el jugador produce una frase de acuerdo con las instrucciones recibidas, sin el apoyo de un modelo que imitar ni de un texto que leer.

La identificación de los saberes más afectados ha de servir a los agentes educativos para proponer unas actividades externas al videojuego que incidan en dichos saberes y así reforzar los beneficios de la intervención educativa. En este sentido, al margen de la enorme potencialidad educativa de los videojuegos, tanto en lo que se refiere al 
aprendizaje indirecto como a la posibilidad de incorporar en ellos dinámicas educativas concretas para la consecución de objetivos particulares, cabe recordar que, como en cualquier campo de la educación, es preciso consolidar el aprendizaje adquirido en el juego mediante el uso de actividades de refuerzo y generalización. En el caso de los alumnos con necesidades específicas de apoyo educativo derivadas de la discapacidad, estas actividades externas al videojuego cobran aun mayor importancia.

Para ello, es esencial la posibilidad que brinda la herramienta THAP-PRADIA de observar la actuación del jugador en cada una de las actividades de forma detallada (serie 14-16) y proponer, si es el caso, ejercicios específicos que repitan las condiciones educativas de la dinámica. A modo de ejemplo, en el caso de (15), la lectura en voz alta de un enunciado con modalidad exhortativa y compuesto de dos grupos prosódicos se puede practicar con otros enunciados del tipo "Lea las noticias y escriba sus comentarios", "Acaba tus tareas y sal a jugar" que remiten a entornos comunicativos distintos del videojuego, y, por tanto, amplían los contextos de uso.

Competencia prosódica / Otras competencias

\begin{tabular}{|l|l|}
\hline Competencia prosódica & 8,31 \\
\hline Otras competencias & 9 \\
\hline
\end{tabular}

Habilidades comunicativas relacionadas con la prosodia

\begin{tabular}{|l|l|}
\hline Competencia prosódica & 8,31 \\
\hline Competencia lingüística emocional & 9,17 \\
\hline Competencia pragmática & 8,18 \\
\hline
\end{tabular}

Ámbitos de la comprensión y la producción

\begin{tabular}{|l|l|}
\hline Discriminación auditiva de estructuras prosódicas & 10 \\
\hline Expresión oral de estructuras prosódicas & 7,79 \\
\hline
\end{tabular}

Categorías entonativas

\begin{tabular}{|l|l|}
\hline Modalidad oracional & 8,8 \\
\hline Agrupación rítmica de enunciados & 7,48 \\
\hline Acentuación enfática & 8,72 \\
\hline
\end{tabular}

Modo de producción oral

\begin{tabular}{|l|l|}
\hline Lectura & 8,01 \\
\hline Imitación & 7,97 \\
\hline Habla elicitada & 7,83 \\
\hline
\end{tabular}

Figura 4. Informe de resultados globales para un jugador en los distintos ámbitos de análisis.

(14) Ejemplo de informe de resultados para una actividad de percepción

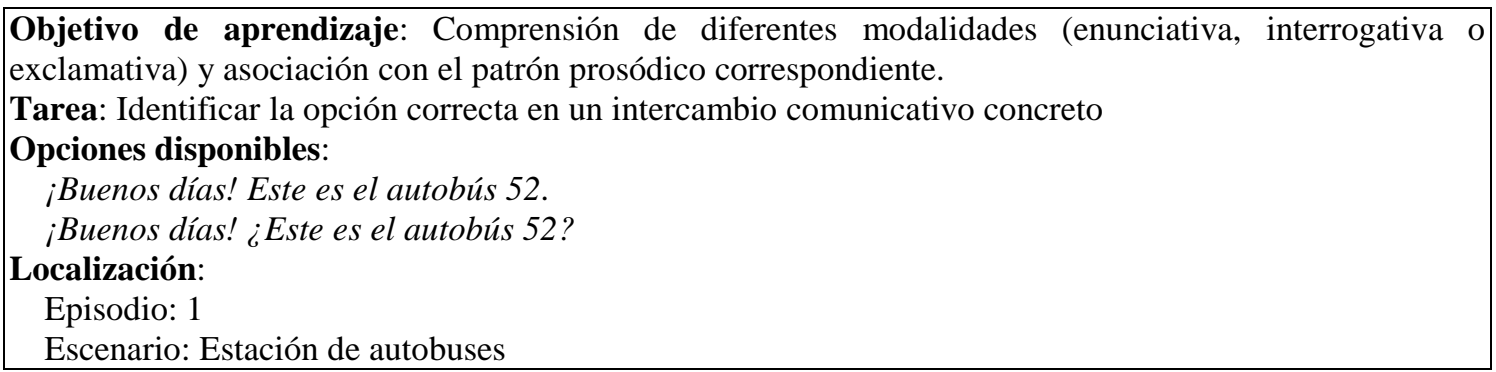


Recuento de errores:

Opciones incorrectas: 0

Competencias trabajadas: Competencia prosódica, Discriminación auditiva de estructuras prosódicas, Modalidad oracional.

(15) Ejemplo de informe de resultados para una actividad de producción

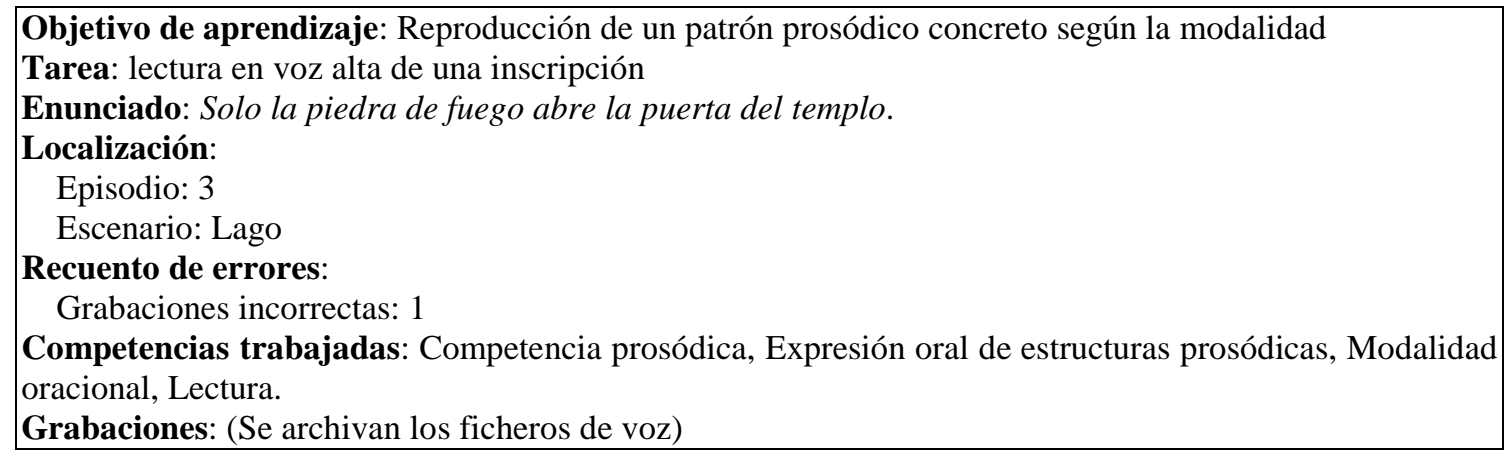

(16) Ejemplo de informe de resultados para una actividad de juego

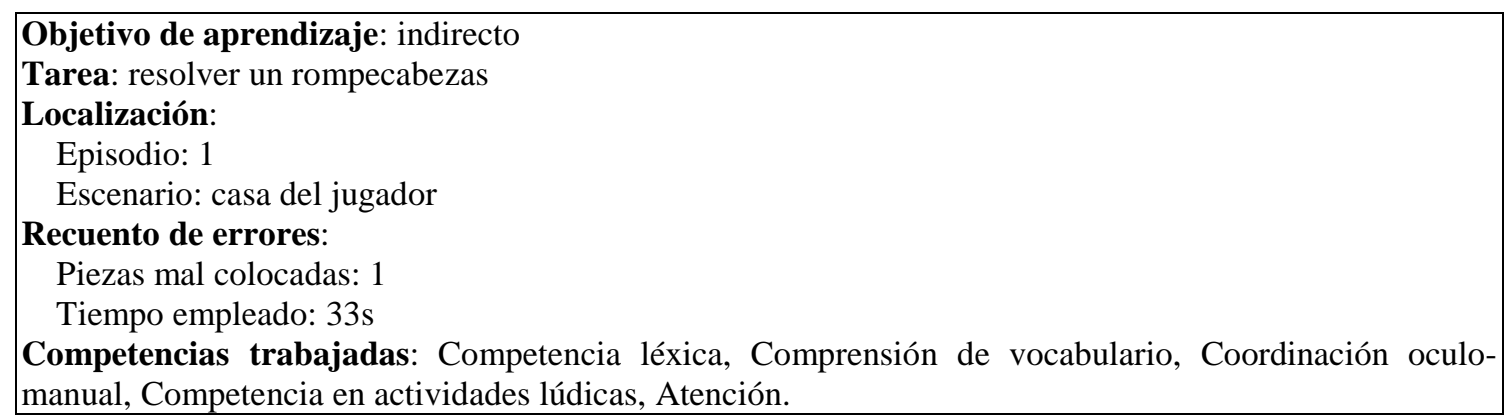

\section{Conclusiones}

En este escrito, una vez expuestas las principales razones para centrarnos en los rasgos prosódicos de la lengua como vía de mejora de la competencia comunicativa de los hablantes con necesidades educativas especiales derivadas de la discapacidad y descritas las características principales del videojuego educativo Pradia: misterio en la ciudad, hemos dirigido la mirada al procedimiento de evaluación que lo acompaña. Cualquier recurso facilitador del aprendizaje, esté o no basado en una tecnología como la del videojuego, debe completarse con un método de evaluación que lo haga eficaz en el contexto educativo. Podemos convenir que los materiales didácticos basados en la tecnología son importantes, pero no tienen un valor especial por sí mismos. Su uso solo queda completamente justificado cuando se integran de forma adecuada en el proceso educativo, de manera que además de contribuir a incrementar la motivación de los estudiantes, les haga aprender de forma duradera.

El Test de habilidades prosódicas del videojuego educativo PRADIA: Misterio en la ciudad (THAP-PRADIA) se concibe como el test post-videojuego de PRADIA: Misterio en la ciudad, un instrumento de evaluación que analiza el grado de éxito en cada una de las actividades resueltas en el videojuego y ofrece unas puntuaciones que estiman el

Instrumento de evaluación de un videojuego educativo facilitador del aprendizaje de habilidades prosódicas y comunicativas. Lourdes Aguilar y Ferran Adell 
progreso en la curva de aprendizaje, con el fin último de identificar logros y carencias de aprendizaje y proponer nuevas estrategias didácticas.

\section{Agradecimientos}

Queremos expresar nuestro agradecimiento a los investigadores que han hecho posible que el trabajo llegue a buen término gracias a su colaboración en diversas etapas del mismo: Mario Corrales, David Escudero, César González-Ferreras y Valle Flores, de la Universidad de Valladolid; Yolanda Martín, del Centro Concertado de Educación Especial "El Pino de Obregón", de Valladolid. La implementación de la aplicación informática ha sido responsabilidad de Mario Corrales, César González-Ferreras y David Escudero.

Presentación del artículo: 26 de abril de 2018

Fecha de aprobación: 9 de julio de 2018

Fecha de publicación: 31 de octubre de 2018

Aguilar, Lourdes y Adell, Ferran (2018). Instrumento de evaluación de un videojuego educativo facilitador del aprendizaje de habilidades prosódicas y comunicativas. RED. Revista de Educación a Distancia, 58. Consultado el (dd/mm/aaaa) en http://www.um.es/ead/red/58/

\section{Financiación}

Este trabajo es parte de un esfuerzo multidisciplinar financiado por la ayuda de la Fundación BBVA a Equipos de Investigación Científica en Humanidades Digitales, 2015. Referencia del proyecto: BBVA2015-01. Cuantía de la subvención: 59.957euros

\section{Referencias bibliográficas}

Aguilar, L. y Gutiérrez-González, Y.M. (en proceso de revisión) Aprendizaje prosódico en un videojuego educativo dirigido a personas con Síndrome de Down: definición de objetivos y diseño de actividades, Revista de Educación Inclusiva.

Aguilar, L., de-la-Mota, C. \& Prieto, P. (coords.) (2009-2014). Guía multimedia de la prosodia del español. Sitio web: http://prado.uab.cat/guia/es/.

Alloza-Castillo, S., Escribano, F., \& Muntada, M. (2017). XBadges. How soft skills are boosted by video games: Improving persistence, risk taking \& spatial reasoning

Instrumento de evaluación de un videojuego educativo facilitador del aprendizaje de habilidades prosódicas y comunicativas. Lourdes Aguilar y Ferran Adell 
with Flappy Bird, Pacman \& Tetris. En 1st Workshop on Gamification and Games for Learning (GamiLearn'17). Universidad de La Laguna.

Canale, M. (1983) "De la competencia comunicativa a la pedagogía comunicativa del lenguaje", en VVAA: Competencia comunicativa. Documentos básicos en la enseñanza de lenguas extranjeras. Madrid: Edelsa, 1995, pp. 63-81.

del Moral, M.E. y Villalustre, L. (2012). Videojuegos e infancia: análisis, evaluación y diseño desde una perspectiva educativa. En García Jiménez; A. (Coord). Comunicación, Infancia y Juventud. Situación e Investigación en España. Barcelona: UOC.

del Moral, M.E., Villalustre, L., Yuste, R., \& Esnaola, G. (2012). Evaluación y diseño de videojuegos: generando objetos de aprendizaje en comunidades de práctica. RED. Revista de Educación a Distancia, (33).

González-Ferreras, C., D. Escudero-Mancebo, M. Corrales-Astorgano, L. Aguilar \& V. Flores-Lucas (2017) Engaging Adolescents with Down Syndrome in an Educational Video Game, International Journal of Human-Computer Interaction, $1-20$.

Granic, I., Lobel, A., \& Engels, R. C. M. E. (2014). The benefits of playing video games. American Psychologist, 69(1), 66-78.

Heselwood, B. C., Bray, M., \& Crookston, I. (1995). Juncture, rhythm and planning in the speech of an adult with Down's syndrome. Clinical Linguistics \& Phonetics, $9(2), 121-137$.

Huizinga, Johan. Homo ludens. Madrid: Alianza, 1984.

Kent, R. D., \& Vorperian, H. K. (2013). Speech impairment in Down syndrome: A review. Journal of Speech, Language, and Hearing Research, 56 (1), 178-210.

Kumin, L. (2002). Inteligibilidad del habla en las personas con Síndrome de Down: Un marco para señalar factores específicos útiles en la evaluación y tratamiento. Rev. Sindr. Down, 14-23.

Ladd, D. R. (2008). Intonational phonology. Cambridge, Cambridge University Press.

Martin, G. E., Klusek, J., Estigarribia, B., \& Roberts, J. E. (2009). Language characteristics of individuals with Down syndrome. Topics in language disorders, 29(2), 112.

Pernía, M. R. G., Gómez, S. C., \& Borda, R. M. (2011). De los videojuegos comerciales al currículum: Las estrategias del profesorado. Revista Icono14, 9(2), 284-299.

Revuelta Domínguez, F. I., \& Guerra Antequera, J. (2012). ¿Qué aprendo con videojuegos? Una perspectiva de meta-aprendizaje del videojugador. RED. Revista de Educación a Distancia, 33.

Rice, M. L., Warren, S. F., \& Betz, S. K. (2005). Language symptoms of developmental language disorders: An overview of autism, Down syndrome, fragile X, specific language impairment, and Williams syndrome. Applied psycholinguistics, 26(01), 7-27. 
Rodríguez-Hoyos, C., \& João Gomes, M. (2013) Videojuegos y educación: Una visión panorámica de las investigaciones desarrolladas a nivel internacional. Profesorado, Revista de Currículum y Formación del Profesorado, 17(2), 479-494.

Stojanovik, V. (2011). Prosodic deficits in children with Down syndrome. Journal of Neurolinguistics, 24(2), 145-155.

Wuang, Y. P., Chiang, C. S., Su, C. Y., \& Wang, C. C. (2011). Effectiveness of virtual reality using Wii gaming technology in children with Down syndrome. Research in developmental disabilities, 32(1), 312-321. 